\title{
Scarlet Fever Upsurge in England and Molecular-Genetic Analysis in North-West London, 2014
}

\section{Claire E. Turner, Marta Pyzio, Bonita Song, Theresa Lamagni, Margie Meltzer, J. Yimmy Chow, Androulla Efstratiou, Sally Curtis, ${ }^{1}$ Shiranee Sriskandan}

Scarlet fever notifications surged across the United Kingdom in spring 2014. Molecular epidemiologic investigation of Streptococcus pyogenes infections in North-West London highlighted increased emm4 and emm3 infections coincident with the upsurge. Unlike outbreaks in other countries, antimicrobial resistance was uncommon, highlighting an urgent need to better understand the drivers of scarlet fever activity.

\begin{abstract}
A $\mathrm{n}$ unprecedented rise in scarlet fever occurred in England in spring 2014, with $>13,000$ notifications, for an overall population rate of $24.5 / 100,000$ persons $(1,2)$. We analyzed clinical notification data for North-West London (population $\approx 1,900,400$ ) during 2009-2014 and determined emm genotypes of Streptococcus pyogenes causing upper respiratory tract (URT) infections during 2009-2014. We focused on peak periods of scarlet fever notification.
\end{abstract}

\section{The Study}

During weeks 10-20 (March-May) 2014, scarlet fever notifications in North-West London increased 3-8-fold compared with the same period in previous years (Figure, panel A). Although Health Protection regulations in England require clinicians to report suspected cases of scarlet fever, molecular surveillance of noninvasive $S$. pyogenes is not feasible because testing for $S$. pyogenes is not routinely advised for patients with a sore throat in the United Kingdom (3). Nonetheless, a limited number of URT swab specimens are submitted by clinicians for culture. Since 2009, we have stored all $S$. pyogenes URT isolates identified in our West London diagnostic laboratory, which serves a population of $\approx 2$ million, overlapping with the North-West London region.

Author affiliations: Imperial College London, London, UK (C.E. Turner, M. Pyzio, S. Sriskandan); Imperial College Healthcare NHS Trust, London (B. Song, S. Curtis); Public Health England, London (T. Lamagni, M. Meltzer, J.Y. Chow, A. Efstratiou)

DOI: http://dx.doi.org/10.3201/eid2206.151726
Molecular testing, with standard DNA extraction, $\mathrm{emm}$ typing, and superantigen typing methods (4), was performed on all 404 viable $S$. pyogenes URT isolates identified. Of all isolates obtained from March through May 2009-2013 (n =72), predominant emm genotypes were emm $12(26 \%)$, emm89 (13\%), and emm 1 (14\%) (Figure, panel B). These proportions were almost identical to proportions for all 308 isolates obtained throughout 2009-2013 (Figure, panel B). In contrast, during March-May $2014(\mathrm{n}=96)$, dominating emm types changed, with a borderline significant increase in emm3 (from 10\% in March-May 2009-2013 to $20 \%$ in $\left.2014 ; \chi_{(1 \mathrm{df})}^{2}=3.766, \mathrm{p}=0.0523\right)$, and a significant $>5$-fold increase in emm 4 (from $3 \%$ to $17 \% ; \chi_{(\text {ldf }}^{2}=9.478$, $\mathrm{p}=0.0021$ ). Among 96 URT samples submitted in MarchMay 2014, a total of 42 were from children ages $\leq 5$ years. $E m m 4$ was significantly associated with age $\leq 5$ years $\left(\chi_{(\text {(dd) }}^{2}\right.$ $=6.046, \mathrm{p}=0.0139$ ), and the rise was largely attributable to disease in this age group (12/17 emm 4 isolates).

Isolates from March-May 2014 were categorized by at least one of the following clinical features (provided by submitting physician): 1) tonsillitis, pharyngitis, or sore throat and no mention of scarlet fever $(n=44) ; 2)$ any mention of scarlet fever, regardless of other information $(\mathrm{n}=$ $16) ; 3)$ any other illness $(n=6)$; and 4$)$ no details provided $(\mathrm{n}=30)$. The 16 scarlet fever-associated isolates were limited to patients ages $1.25-11$ years, a significant proportion of whom were $\leq 5$ years $\left(12 / 16 ; \chi_{(1 \mathrm{df})}^{2}=7.619, \mathrm{p}=0.0058\right)$; $7 / 16$ were emm 3 and $3 / 16$ were emm 4 . The remainder were emm 12 (3), emm 28 (2), and emm87 (1). On the basis of these limited data, emm 3 was significantly associated with scarlet fever in $2014\left(\chi_{(1 \mathrm{df})}^{2}=5.964, \mathrm{p}=0.0146\right)$.

Clinical data were not collected in earlier years routinely, although in 2009 a total of 3/3 isolates from scarlet fever case-patients were emm3. Scarlet fever-associated $\mathrm{emm} 4$ strains from $2014(\mathrm{n}=3)$ carried superantigens speC, ssa, and smeZ; the same superantigen profile was found in emm 4 strains from patients for whom scarlet fever was not mentioned $(\mathrm{n}=14)$. All 7 scarlet fever-associated emm3 strains carried speA, ssa, speG, and a known mutation in smeZ.

Antimicrobial drug resistance was identified in 10/96 URT isolates from 2014; however, none of these isolates were associated with scarlet fever and none were emm 4 .

${ }^{1}$ Current affiliation: Royal Sussex County Hospital, Brighton, UK. 
Figure. Increase in North-West London scarlet fever notifications and association with emm4 and emm3, 2014. A) Weekly scarlet fever notifications in North-West London during 2009-2014. During weeks 10-20 (March-May) 2014 , the number of notifications substantially increased. B) emm genotyping of 404 upper respiratory tract Streptococcus pyogenes isolates. Isolates were available from March 2009 through May 2014, inclusive. A total of 308 isolates were from 2009-2013; however, of these, 134 were from 2009 , and 174 were from 20102013 , reflecting a fall in submission rates and affecting the availability of strains for study. Thus, isolates from 2009-2013 were considered a single group (JanuaryDecember) and the following emm-types were identified: emm12 $20 \%$ (62/308), emm89 16\% (48/308), emm1 15\% (47/308), emm3 7\% (22/308), emm28 6\% (19/308), emm4 3\% (10/308).

A similar pattern was observed during March-May in 2009-2013 $(n=72)$. A total of 96 isolates were available from 2014 , all of which were from March-May, submitted following alerts to clinicians regarding scarlet fever activity. During March-May 2014, isolates typed as emm4 increased significantly, from 3\% (2/72) to 17\% $(17 / 96),\left(\chi_{(1 \mathrm{df})}^{2}=9.478 . p=0.0021\right)$. A borderline significant increase occurred in emm3, from $10 \%(7 / 72)$ in $2009-2013$ to $20 \%(20 / 96)$ in $2014\left(\chi_{(1 \mathrm{df})}^{2}=3.766, p=0.0523\right)$. This constituted a 3-fold increase in emm3 and emm4 combined, from 13\% in 2009-2013 to 37\% in 2014 (March-May).

Erythromycin resistance was found in $2 / 20$ non-scarlet fever emm 3 isolates, in combination with clindamycin resistance in 1 isolate.

\section{Conclusions}

An increase in emm 3 and emm 4 S. pyogenes URT isolates was detected in North-West London, during the period in 2014 when scarlet fever notifications peaked. The increase in emm 4 infections was also found predominantly in 4- to 5-year-old children, the group we and others found to be most at risk for scarlet fever (1). The percentage of children 4 years old in North-West London (an urban population) is similar to the national average of $1.3 \%$; therefore, our findings are probably relevant to the rest of the United Kingdom.

Emm 4 isolates accounted for only 3/16 cases in which scarlet fever was mentioned, although, because of the study's retrospective nature and paucity of clinical data supplied, we cannot dismiss the possibility that other emm 4 isolates were also associated with scarlet fever. On the basis of the limited analysis of isolates from infections in which scarlet fever was mentioned, we found an association between scarlet fever and $S$. pyogenes emm 3 strains.

The results of our historical comparison must be interpreted with caution; obtaining swab samples from patients with URT infections in England is not routine. Thus, the 2009-2013 samples may reflect persistent infections, in contrast to 2014 samples, when clinicians were encouraged to submit swab specimens for scarlet fever case-patients. Furthermore, the number of strains available for emm typing was limited. Nonetheless, this was the only collection of strains available to us that permitted historical comparison.

Both emm 3 and emm 4 S. pyogenes strains have been associated with scarlet fever (5). In the Far East, emm 1 and emm4 isolates were the leading causes of scarlet fever in the late 1990s (6), although more recently, antimicrobial 
drug-resistant emm12 S. pyogenes has dominated in this region (7-9). We found that the proportion of emm 12 isolates fell during the scarlet fever surge and found no antimicrobial drug resistance among emm 3 or emm 4 isolates associated with scarlet fever.

Emm4 isolates are associated with pharyngitis in children $(10,11)$; these isolates are entirely acapsular, a phenotype linked to enhanced adhesion to surfaces (12). Whether this characteristic can increase persistence and transmission is unknown. Surges in scarlet fever are believed to require a population susceptible to pharyngeal infection with specific strain types and specific superantigens. Both emm 3 and emm 4 strains in our study possessed 2 prophage-associated superantigens, either SPEA and SSA, or SPEC and SSA. Although these toxin genes were found in emm 3 and emm 4 strains not associated with scarlet fever, the probability of triggering scarlet fever may be enhanced through production of 2 such superantigens. An association between these superantigens and scarlet fever has been reported (13).

Periodic increases in scarlet fever are well recognized, although the magnitude of the upsurge in the United Kingdom was unexpected. Consultation rates for sore throat diminished in the 1990s (14), and the 2008 UK national guidelines advise against diagnostic testing and recommend a policy of nonprescribing or delayed prescribing for sore throat when the Centor score is $<3$ (3). These recommendations contrast with those of North America and of some European countries (15). Whether exceeding a threshold level of community $S$. pyogenes transmission is required for such a marked upsurge is unclear; increased scarlet fever activity was not reported elsewhere in Europe, to our knowledge. Apart from natural fluctuations in population immunity, emergence of hypertransmissible lineages, acquisition of novel phage-encoded toxins, or antimicrobial drug resistance may contribute to scarlet fever surges $(6,7)$. Notably, isolates we found associated with scarlet fever were not resistant to common antimicrobial agents.

As part of the national response, clinicians were advised to treat scarlet fever to minimize complications and reduce transmission. Whether use of more refined molecular diagnostics could assist future community prevention and management of $S$. pyogenes infection will require careful evaluation. Increased scarlet fever activity has continued in England in 2015 and 2016, underscoring the need for ongoing surveillance and further investigation.

\section{Acknowledgments}

We are grateful to Hugo Donaldson and staff of the local diagnostic laboratory of Imperial College Healthcare National Health Service Trust, who performed identification and susceptibility testing, and to the clinical staff, who reported cases of scarlet fever.
This project was funded by the National Institute for Health Research Biomedical Research Centre awarded to Imperial College Healthcare NHS Trust and the UK Clinical Research Collaboration (UKCRC, Centre for Infection Prevention and Management).

Dr. Turner is a Junior Research Fellow in the Department of Medicine, Imperial College London. Her primary research interests are streptococcal pathogenesis and bacterial population genomics.

\section{References}

1. Guy R, Williams C, Irvine N, Reynolds A, Coelho J, Saliba V, et al. Increase in scarlet fever notifications in the United Kingdom, 2013/2014. Euro Surveill. 2014;19:20749. http://dx.doi.org/10.2807/1560-7917.ES2014.19.12.20749

2. Public Health England. Group A streptococcal infections: seasonal activity, 2014/15. Health Protection Report. 2014;8 [cited 2015 Sep 28]. https://www.gov.uk/government/uploads/system/uploads/ attachment_data/file/377520/hpr4414_SF.pdf

3. National Institute for Health and Care Excellence. Respiratory tract infections: antibiotic prescribing. Prescribing antibiotics for self-limiting respiratory tract infections in adults and children in primary care (NICE guideline), July 2008 [cited 2015 Sep 28]. https://www.nice.org.uk/guidance/CG69/chapter/1-Guidance

4. Turner CE, Dryden M, Holden MT, Davies FJ, Lawrenson RA, Farzaneh L, et al. Molecular analysis of an outbreak of lethal postpartum sepsis caused by Streptococcus pyogenes. J Clin Microbiol. 2013;51:2089-95. http://dx.doi.org/10.1128/JCM.00679-13

5. Perks EM, Mayon-White RT. The incidence of scarlet fever. J Hyg Camb. 1983;91:203-9. http://dx.doi.org/10.1017/S0022172400060204

6. Yan JJ, Liu CC, Ko WC, Hsu SY, Wu HM, Lin YS, et al. Molecular analysis of group A streptococcal isolates associated with scarlet fever in southern Taiwan between 1993 and 2002. J Clin Microbiol. 2003;41:4858-61. http://dx.doi.org/10.1128/ JCM.41.10.4858-4861.2003

7. Tse H, Bao JY, Davies MR, Maamary P, Tsoi HW, Tong AH, et al. Molecular characterization of the 2011 Hong Kong scarlet fever outbreak. J Infect Dis. 2012;206:341-51. http://dx.doi.org/10.1093/ infdis/jis 362

8. Chiou CS, Wang YW, Chen PL, Wang WL, Wu PF, Wei HL. Association of the shuffling of Streptococcus pyogenes clones and the fluctuation of scarlet fever cases between 2000 and 2006 in central Taiwan. BMC Microbiol. 2009;9:115. http://dx.doi.org/ 10.1186/1471-2180-9-115

9. Luk EYY, Lo JYC, Li AZL, Lau MCK, Cheung TKM, Wong AYM, et al. Scarlet fever epidemic, Hong Kong, 2011. Emerg Infect Dis. 2012;18:1658-1661. http://dx.doi.org/10.3201/eid1810.111900

10. Jaggi P, Tanz RR, Beall B, Shulman ST. Age influences the emm type distribution of pediatric group A streptococcal pharyngeal isolates. Pediatr Infect Dis J. 2005;24:1089-92. http://dx.doi.org/ 10.1097/01.inf.0000190023.89759.96

11. Shulman ST, Tanz RR, Dale JB, Beall B, Kabat W, Kabat K, et al. Seven-year surveillance of North American pediatric group A streptococcal pharyngitis isolates. Clin Infect Dis. 2009;49:78-84. http://dx.doi.org/10.1086/599344

12. Turner CE, Abbott J, Lamagni T, Holden MT, David S, Jones MD, et al. Emergence of a new highly successful acapsular group A Streptococcus clade of genotype emm 89 in the United Kingdom. MBio. 2015;6:e00622. http://dx.doi.org/10.1128/mBio.00622-15

13. Silva-Costa C, Carrico JA, Ramirez M, Melo-Cristino J. Scarlet fever is caused by a limited number of Streptococcus pyogenes lineages and is associated with the exotoxin genes ssa, speA and 
speC. Pediatr Infect Dis J. 2014;33:306-10. http://dx.doi.org/ 10.1097/INF.0000000000000088

14. Ashworth M, Latinovic R, Charlton J, Cox K, Rowlands G, Gulliford M. Why has antibiotic prescribing for respiratory illness declined in primary care? A longitudinal study using the General Practice Research Database. J Public Health (Oxf). 2004;26:268-74. http://dx.doi.org/10.1093/pubmed/fdh160

15. Chiappini E, Regoli M, Bonsignori F, Sollai S, Parretti A, Galli L, et al. Analysis of different recommendations from international guidelines for the management of acute pharyngitis in adults and children. Clin Ther. 2011;33:48-58. http://dx.doi.org/10.1016/ j.clinthera.2011.02.001

Address for correspondence: Shiranee Sriskandan, Infectious Diseases and Immunity, Department of Medicine, Imperial College London, Hammersmith Hospital, Du Cane Rd, London, W12 0NN, UK; email: s.sriskandan@imperial.ac.uk

\section{EMERGING INFECTIOUS DISEASES}

\section{JOURNAL BACKGROUND AND GOALS}

\section{What are "emerging" infectious diseases?}

Infectious diseases whose incidence in humans has increased in the past 2 decades or threatens to increase in the near future have been defined as "emerging." These diseases, which respect no national boundaries, include

$\star$ New infections resulting from changes or evolution of existing organisms.

$\star$ Known infections spreading to new geographic areas or populations.

* Previously unrecognized infections appearing in areas undergoing ecologic transformation.

$\star$ Old infections reemerging as a result of antimicrobial resistance in known agents or breakdowns in public health measures.

\section{Why an "Emerging" Infectious Diseases journal?}

The Centers for Disease Control and Prevention (CDC), the agency of the U.S. Public Health Service charged with disease prevention and health promotion, leads efforts against emerging infections, from AIDS, hantavirus pulmonary syndrome, and avian flu, to tuberculosis and West Nile virus infection. CDC's efforts encompass improvements in disease surveillance, the public health infrastructure, and epidemiologic and laboratory training.

Emerging Infectious Diseases represents the scientific communications component of CDC's efforts against the threat of emerging infections. However, even as it addresses CDC's interest in the elusive, continuous, evolving, and global nature of these infections, the journal relies on a broad international authorship base and is rigorously peer-reviewed by independent reviewers from all over the world.

\section{What are the goals of Emerging Infectious Diseases?}

1) Recognition of new and reemerging infections and understanding of factors involved in disease emergence, prevention, and elimination. Toward this end, the journal

* Investigates factors known to influence emergence: microbial adaptation and change, human demographics and behavior, technology and industry, economic development and land use, international travel and commerce, and the breakdown of public health measures.

$\star$ Reports laboratory and epidemiologic findings within a broader public health perspective.

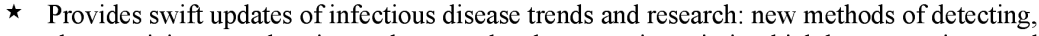
characterizing, or subtyping pathogens; developments in antimicrobial drugs, vaccines, and prevention or elimination programs; case reports.

2) Fast and broad dissemination of reliable information on emerging infectious diseases. Toward this end, the journal

$\star$ Publishes reports of interest to researchers in infectious diseases and related sciences, as well as to public health generalists learning the scientific basis for prevention programs.

$\star$ Encourages insightful analysis and commentary, stimulating global interest in and discussion of emerging infectious disease issues.

* Harnesses electronic technology to expedite and enhance global dissemination of emerging infectious disease information. 